This document is confidential and is proprietary to the American Chemical Society and its authors. Do not copy or disclose without written permission. If you have received this item in error, notify the sender and delete all copies.

\title{
Complex dynamics of a fluorinated vinylidene cyanide copolymer highlighted by dielectric relaxation spectroscopy
}

\begin{tabular}{|r|l|}
\hline Journal: & Macromolecules \\
\hline Manuscript ID & ma-2016-006839.R1 \\
\hline Manuscript Type: & Article \\
\hline Date Submitted by the Author: & n/a \\
\hline Complete List of Authors: & $\begin{array}{l}\text { Castelvetro, Valter; University of Pisa, Department of Chemistry and } \\
\text { Industrial Chemistry } \\
\text { Capaccioli, Simone; Università di Pisa, Physics } \\
\text { Raihane, Mustapha; Univeristy of Marrakech, Laboratoire de Chimie } \\
\text { Bioorganique et Macromoléculaire } \\
\text { Atlas, Salima; Cadi Ayyad University, Faculty of Sciences and Techniques, } \\
\text { Laboratory of Organometallic and Macromolecular Chemistry- Composite } \\
\text { Materials }\end{array}$ \\
\hline
\end{tabular}




\title{
Complex dynamics of a fluorinated vinylidene
}

\section{cyanide copolymer highlighted by dielectric}

\section{relaxation spectroscopy}

\author{
Valter Castelvetro, ${ }^{1, *}$ Simone Capaccioli, ${ }^{2}$ Mustapha Raihane, ${ }^{3}$-Atlas Salima ${ }^{3}$ \\ ${ }^{1}$ Dipartimento di Chimica e Chimica Industriale, Università di Pisa, via G. Moruzzi 13, I-56124, \\ Pisa, Italy \\ ${ }^{2}$ Dipartimento di Fisica, Università di Pisa, Largo Pontecorvo 3, I-56127 Pisa, Italy \\ ${ }^{3}$ Laboratory of Organometallic and Macromolecular Chemistry- Composite Materials, Faculty \\ of Sciences and Techniques, Avenue Abdelkrim Khattabi, BP 549, 40000 Marrakech, Morocco
}

\begin{abstract}
The complex dynamics of a nearly alternating copolymer of vinylidene cyanide (1,1-dicyanoethylene, VCN) with 2,2,2-trifluoroethyl methacrylate (TFEMA), including two $\alpha$ relaxations with diverging time scale in the glass transition temperature range, was thoroughly characterized by dielectric spectroscopy over wide temperature and frequency ranges and analysed in the frame of the Ngai's Coupling Model. The dielectric relaxation strength as well as the glass transition temperature, the temperature dependence of the $\alpha$-relaxation time and the corresponding distribution of relaxation times were all larger than those of a reference TFEMA
\end{abstract}


homopolymer, as expected from the introduction of the stiffening VCN units all along the macromolecular chain. The effect of casting solvent and applied poling electric field on the copolymer dielectric strength suggests the onset of local orientational order involving the strong dipoles in the VCN units, a requirement for piezo-and pyroelectricity in amorphous polymers.

KEYWORDS. 2,2,2-trifluoroethyl methacrylate; vinylidene cyanide; dielectric spectroscopy; amorphous copolymer; coupling model; piezoelectricity.

\section{Introduction}

Polymers containing highly polar substituents are of potential interest for the development of advanced electrical and optical materials. In particular, if an average net polarization deriving from spatially oriented structural domains can be achieved by e.g. mechanical stretching or electrical poling, such polymers may display macroscopic piezoelectric, pyroelectric and/or ferroelectric properties. ${ }^{1}$ Piezoelectricity of organic materials was first discovered in the semicrystalline homopolymer of vinylidene fluoride (PVDF) and its copolymer with trifluoroethylene. ${ }^{2,3}$ Their piezoelectricity arises from the large dipole moments generated upon mechanically or electrically induced orientation of the crystalline domains, causing the transition to non-centrosymmetric structures such as the crystalline $\beta$ phase of all-trans PVDF. Such polarization is preserved for a long time at room temperature even if the crystal domains are embedded in an amorphous phase characterized by a low glass transition temperature $\left(\mathrm{T}_{\mathrm{g}}=238 \mathrm{~K}\right.$ for PVDF). However, to explain the presence of a Curie temperature below the melting point in 
PVDF and its copolymers, piezoelectricity has been also associated with Coulomb interactions between injected, trapped charges and oriented dipoles in the crystals. ${ }^{4}$

As a corollary to the above, also amorphous polymers can exhibit piezoelectricity and pyroelectricity. However, in amorphous polymers the polarization is not in a state of thermal equilibrium, but rather a metastable state in which strong molecular dipoles are aligned and frozen into a glass. The net polarization required to provide macroscopic piezo- or pyroelectric properties can only be achieved after a poling procedure carried out at or above $\mathrm{T}_{\mathrm{g}}$ and capable of inducing a non-centrosymmetric orientation of the dipoles. The effectiveness of the procedure, that is the fraction of dipoles eventually frozen in the glassy state parallel to the field direction, and thus the value of dielectric strength $\Delta \varepsilon$, i.e. the fraction of static permittivity due to the orientation of permanent dipoles, depend on both the magnitude of the microstructural dipole moments and the distribution of the corresponding polar groups along the copolymer chain. In a copolymer an even distribution of polar groups is more easily achieved if the structure is regularly alternating, although the tacticity may also play an important role. Furthermore, it is the $\mathrm{T}_{\mathrm{g}}$ value that ultimately defines the temperature application range; in fact an amorphous piezoelectric polymer must be used in its glassy state and possibly well below $\mathrm{T}_{\mathrm{g}}$. While the limited ability to strain under an applied stress typical of any stiff glassy material is an significant drawback, piezoelectricity in amorphous polymers remains a very interesting feature owing to the broader range of structures and properties available, which can result in broader application versatility.

Among the amorphous polymers investigated for their piezoelectric behaviour, cyano (nitrile) containing polymers play a particularly important role. Since the discovery of the piezoelectric activity of the alternating copolymer of vinylidene cyanide (1,1-dicyanoethylene, VCN) with 
vinyl acetate, ${ }^{5,6}$ there has been growing interest in the synthesis and characterization of homoand copolymers of nitrile monomers with various vinyl comonomers such as vinyl esters, styrene, acrylic and methacrylic esters, as well as their functional derivatives. ${ }^{7-16,}$ The microstructure of such polymers has been investigated in relation to their nonlinear dielectric behaviour $^{15-17}$ and their dielectric strength, $\Delta \varepsilon$, above $T_{g}$ which was found in some cases to be extraordinarily large, leading to significant piezo- and pyroelectricity.

On the other hand, despite the well-known nonlinear dielectric behaviour of fluorinated polymers such as PVDF and other semicrystalline fluoroolefin copolymers, the dielectric properties of amorphous fluorinated polymers such as those based on fluoroalkyl acrylates and methacrylates have rarely been investigated. S. Koizumi et al. ${ }^{18}$ studied a series of poly(fluoroalkyl methacrylate)s and assigned the observed $\alpha$ and $\gamma$ relaxations to segmental reorientation and local molecular motion of fluoroalkyl side groups above and below $\mathrm{T}_{\mathrm{g}}$, respectively. More recently, a series of amorphous copolymers of 2,2,2-trifluoroethyl methacrylate (TFEMA, also named in the literature with the acronym MATRIF) and 2,2,2trifluoroethyl acrylate with methacrylonitrile (MAN), acrylonitrile (AN), and methylvinylidene cyanide (MVCN) were synthesized by free radical polymerization and their microstructural, thermal and dielectric properties investigated. ${ }^{19-21}$ As a further expansion of this investigation on the synthesis and characterization of new dielectric polymers containing cyano and fluoroalkyl substituents with strong dipole moments, a nearly alternating copolymer of TFEMA with VCN, poly(VCN-co-TFEMA), was also synthesized and its microstructural features and thermal degradation behaviour were investigated. ${ }^{22,23}$. In the present paper the results of a thorough characterization of poly(VCN-co-TFEMA) by means of dielectric spectroscopy are presented and compared with those of a reference TFEMA homopolymer, poly(TFEMA), to highlight the 
significant changes introduced by the incorporation of the rigid and polar VCN comonomer. In particular, the $\alpha$ - and $\beta$-relaxation dynamics is analysed in the frame of the Ngai's coupling model $^{24}$ while a possible correlation between the polymer structure and its dielectric strength, and hence its potential piezoelectric response, is critically discussed.

\section{Experimental Section}

\section{Materials}

The synthesis and a detailed structural characterization of poly(VCN-co-TFEMA) have been reported elsewhere. ${ }^{22}$ The copolymer was prepared according to the scheme of Fig.1 by bulk free radical polymerization using azobis-isobutirronitrile (AIBN) as the initiator and an equimolar comonomer feed, resulting in a final composition richer in the fluorinated comonomer (VCN/TFEMA $=42 / 58 \mathrm{~mol} / \mathrm{mol})$. The copolymer, with molecular weight $\overline{M_{n}}=19,000 \mathrm{~g} \cdot \mathrm{mol}^{-1}$ and polydispersity $\overline{M_{w}} / \overline{M_{n}}=2.3$ (by size exclusion chromatography) is soluble in DMF and moderately soluble in DMSO, chloroform, and acetonitrile. High resolution ${ }^{1} \mathrm{H}$ and ${ }^{13} \mathrm{C}$ NMR spectra had shown a comonomer sequence distribution in which the presence of VCN homodyads or longer homosequences is negligible. The amorphous copolymer, with glass transition at $\mathrm{T}_{\mathrm{g}}=373 \mathrm{~K}$ (midpoint of the heat capacity jump; $368 \mathrm{~K}$ from the onset, as determined by differential scanning calorimetry), shows a thermal stability significantly improved with respect to the two parent homopolymers, the onset of its main pyrolytic degradation step occurring at $\mathrm{T}_{\mathrm{d}}=641 \mathrm{~K}^{23} \mathrm{~A}$ reference TFEMA homopolymer, poly(TFEMA), was also synthesized by free radical polymerization in dioxane initiated by $1 \mathrm{wt} \%$ azobis-isobutirronitrile (AIBN) $\left(\overline{M_{n}}=20,000 \mathrm{~g} \cdot \mathrm{mol}^{-1}\right.$, polydispersity $\overline{M_{w}} / \overline{M_{n}}=2.6 ;$ onset of the first pyrolytic decomposition step at $\left.\mathrm{T}_{\mathrm{d}}=519 \mathrm{~K}\right)$. DSC analysis confirmed also for the homopolymer the 
expected amorphous structure, with glass transition temperature $\mathrm{T}_{\mathrm{g}}=341 \mathrm{~K}$ (midpoint; $\mathrm{T}_{\mathrm{g}}=334 \mathrm{~K}$ from the onset of the heat capacity jump).

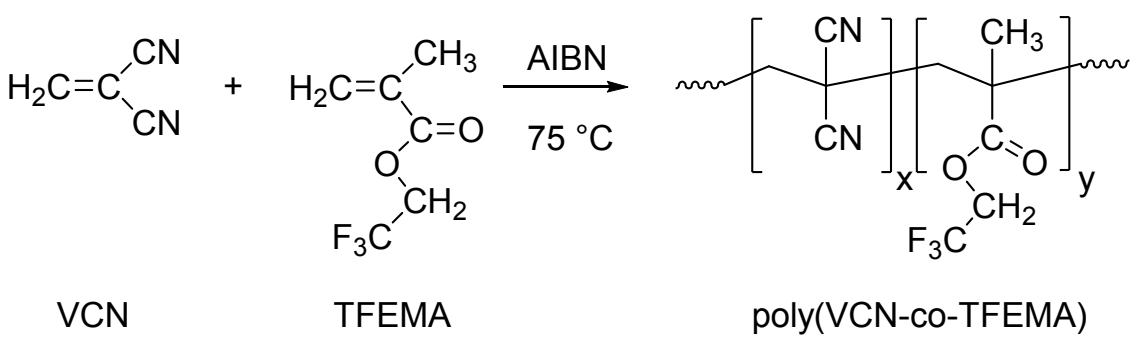

Figure 1. Synthetic scheme for poly(VCN-co-TFEMA) $(\mathrm{x}=42, \mathrm{y}=58)$

\section{Dielectric Spectroscopy setup and methods}

The polymer films were obtained from solutions in the appropriate solvent (acetonitrile or chloroform) by casting onto a flat circular gold plated electrode (diameter $30 \mathrm{~mm}$ ). The films were allowed to dry in air, then under a vacuum to remove any residual solvent. The thickness of the film, as determined by a profilometer, was always uniform and in the $100-120 \mu \mathrm{m}$ range. A gold layer was evaporated onto the top side of the film to ensure a good electric contact. The coated film was sandwiched between two gold plated electrodes and conditioned in vacuum at about $30 \mathrm{~K}$ above $T_{g}$ under a moderate pressure to ensure good and uniform electrical contacts.

The geometric capacitance $\mathrm{C}_{0}$ of the parallel plate capacitors was in the 50-60 $\mathrm{pF}$ range. The complex capacitance $C(\omega)=C^{\prime}(\omega)-j C^{\prime \prime}(\omega)$ of the samples was measured under isothermal conditions at different frequencies $f=\omega / 2 \pi(0.4 \mathrm{mHz} \leq f \leq 10 \mathrm{MHz})$ by means of a Novocontrol Dielectric Analyser to obtain the complex permittivity $\varepsilon=\varepsilon^{\prime}-\mathrm{j} \varepsilon^{\prime \prime}=\mathrm{C}(\omega) / \mathrm{C}_{0}$, and thus the values of real, $\varepsilon^{\prime}$, and imaginary, $\varepsilon^{\prime \prime}$, part of permittivity as well as the tangent of loss angle $\tan \delta=\varepsilon^{\prime \prime} / \varepsilon^{\prime}$.

The sample was stabilized at the target temperature for $20 \mathrm{~min}$ before running the measurements at different frequencies, keeping the temperature stable within $0.1 \mathrm{~K}$. The 
temperature range between $120 \mathrm{~K}$ and $450 \mathrm{~K}$, spanning well below and above the glass transition temperature, was investigated. In the case of the reference homopolymer, poly(TFEMA), the upper temperature limit was $415 \mathrm{~K}$. Two different procedures were applied: first the sample was heated to the upper temperature limit and cooled down to the lower one at subsequent steps, equilibrating at each step before performing the dielectric measurements as mentioned before. Then the sample was heated up again following the same steps and equilibration and a second series of measurements, each one at a progressively increasing temperature, was carried out. No significant differences between the dielectric properties obtained during the two runs were found.

\section{Results and Discussion}

The molecular dynamics of poly(VCN-co-TFEMA) and of the parent homopolymer, poly(TFEMA), have been investigated by broadband dielectric spectroscopy as a function of temperature. The results of the characterization of poly(TFEMA), intended here as a reference material lacking the highly polar and structurally rigid VCN unit of the copolymer, are presented beforehand, along with the theoretical background and the models adopted to fit the experimental data throughout this work.

\subsection{Dynamics of poly(TFEMA)}

Poly(TFEMA) exhibits a dynamic scenario with 3 relaxation processes, similarly to that shown by its unfluorinated analogous poly(ethyl methacrylate), poly(EMA) ${ }^{25}$ A summary of the overall relaxation behavior is shown in Fig. 2 in which selected spectra of isothermal scan as a function of frequency are reported along with the respective fitting functions (isochronal scans at $v=1 \mathrm{~Hz}$, $117 \mathrm{~Hz}$ and $10 \mathrm{kHz}$, respectively, as a function of temperature were also recorded and can be found in Fig. S1 of the Supporting Information). In the isothermal relaxation spectra the $\alpha$ - 
process accounts for the quite intense relaxation dominating $\varepsilon^{\prime}$ (Fig. 2a), shifting to lower frequencies on decreasing temperature and detectable down to around $340 \mathrm{~K}$, with a corresponding behavior displayed by the broad peak in dielectric loss $\varepsilon^{\prime \prime}$ (Fig. 2b).

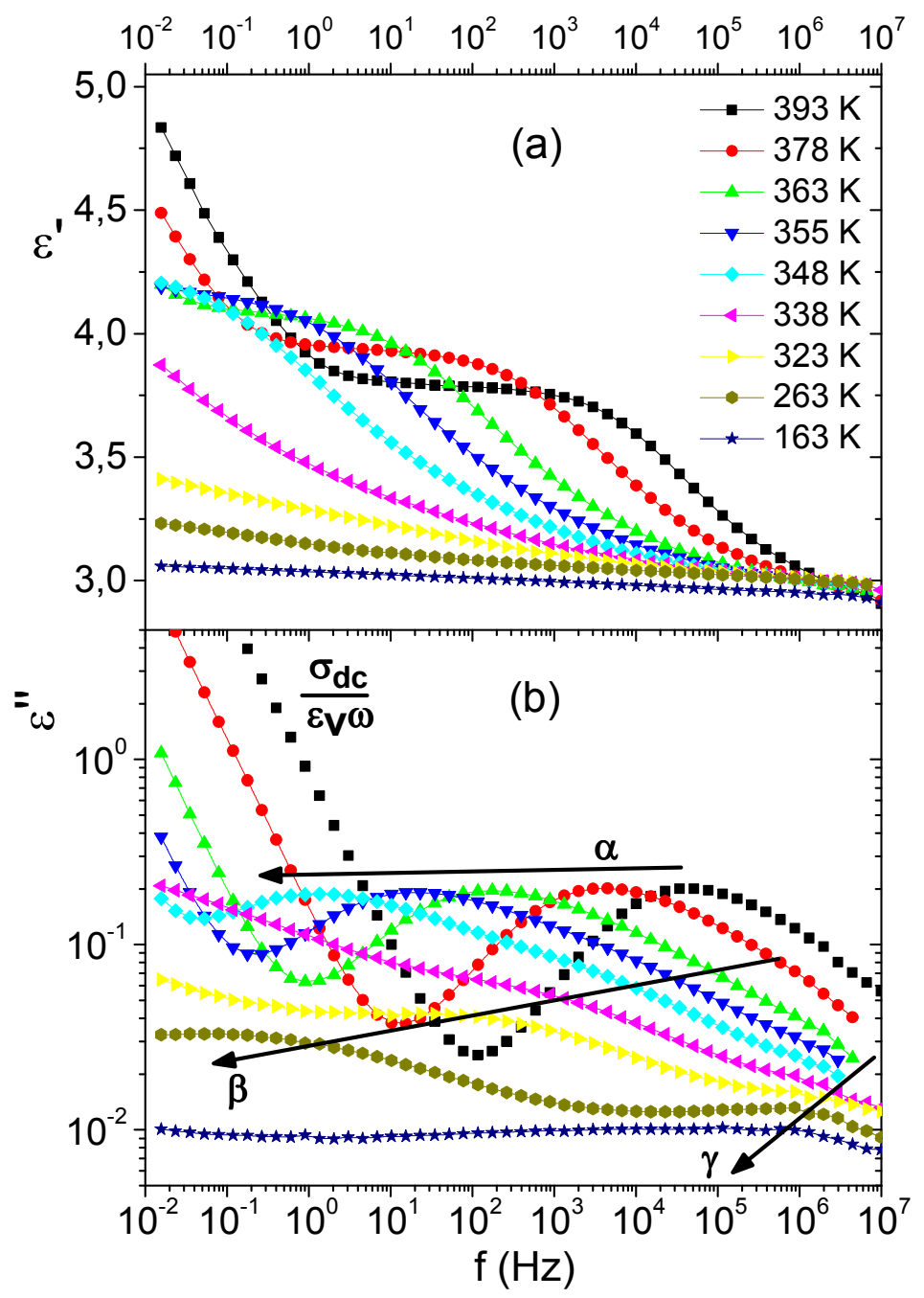

Figure 2. Selected isothermal spectra of poly(TFEMA) in the 393-163 K temperature range: (a) real part of permittivity ( $\left.\varepsilon^{\prime}\right)$; (b) imaginary part (dielectric loss, $\varepsilon^{\prime \prime}$ ). 
Additionally, in the low frequency part of $\varepsilon^{\prime \prime}$ the characteristic contribution of d.c. conductivity, $\sigma_{\mathrm{dc}}$ (reciprocal of frequency), due to drift of ionic impurities can be observed (Fig. 2b). At $T<T_{g}$ two secondary relaxation processes, $\beta$ and $\gamma$, are clearly detectable. More details about molecular assignments of these relaxation process are discussed in the Supporting Information (Fig. S2) and later in this paper, together with the relaxation map (Fig. 3).

A simultaneous fit of the real and imaginary parts of permittivity was used to analyse the isothermal dielectric spectra. The fitting function is a sum of the d.c. conductivity contribution and of two relaxation processes ( $\alpha$ and $\beta$ above $\mathrm{T}_{\mathrm{g}}, \beta$ and $\gamma$ below $\mathrm{T}_{\mathrm{g}}$ ), represented by a superposition of Havriliak-Negami (HN) functions. ${ }^{26}$

$\varepsilon=\varepsilon_{\infty}+\sum_{i} \frac{\Delta \varepsilon_{i}}{\left(1+\left(j \omega \tau_{H N_{i}}\right)^{1-a_{i}}\right)^{b_{i}}}+j \frac{\sigma_{d c}}{\varepsilon_{V} \omega}$

with $\mathrm{i}=\alpha-, \beta$-, and $\gamma$-relaxations, respectively, and where $\tau_{H N i}$ is the $\mathrm{HN}$ relaxation time parameter, $\Delta \varepsilon_{\mathrm{i}}$ is the dielectric relaxation strength, $\mathrm{a}_{\mathrm{i}}$ and $\mathrm{b}_{\mathrm{i}}$ are the $\mathrm{HN}$ shape parameters, $\varepsilon_{V}$ is the vacuum permittivity and $j$ is the imaginary unit. The different contributions, as well as the value of the high frequency permittivity, $\varepsilon_{\infty}$, have been singled out by the fitting procedure. Further details on the appropriateness of the HN functions for the fitting procedure are reported in the Supporting Information (Fig. S3).

The molecular dynamics features can be described by the most probable relaxation time, i.e. $\tau_{\max }=1 /\left(2 \pi f_{\max }\right)$, that was taken as the characteristic relaxation time for each relaxation process, where $f_{\max }$ is the frequency of the peak maximum, calculated from the fitting $\mathrm{HN}$ equations according to the relation:

$$
f_{\max }=\frac{1}{2 \pi \tau_{H N}}\left[\frac{\sin \frac{\pi(1-a)}{2(1-b)}}{\sin \frac{\pi(1-a) b}{2(1+b)}}\right]^{1 /(1-a)}
$$


Another important parameter is the so-called dielectric increment or total static dielectric polarization $\Delta \varepsilon_{\mathrm{T}}$, that can be obtained as the difference between the static permittivity $\left(\varepsilon_{\mathrm{S}}\right)$ and $\varepsilon_{\infty}$ or by the sum of the dielectric relaxation strengths associated with the various relaxation processes. According to the theory of Onsager, ${ }^{27}$ this parameter is proportional to the effective number of dipoles aligned with the external applied field:

$\Delta \varepsilon_{T}=\varepsilon_{S}-\varepsilon_{\infty}=\sum_{i} \Delta \varepsilon_{i}=\frac{g_{k}}{k T} \cdot \sum_{j} N_{j} \mu_{j}^{2}$

where $\mu_{\mathrm{j}}$ is the dipole moment associated to the molecular group $\mathrm{j}, \Sigma_{\mathrm{j}} \mathrm{N}_{\mathrm{j}}$ is the total volume concentration of molecular dipoles, and $\mathrm{g}_{\mathrm{k}}$ is the Kirkwood factor that takes into account the relative molecular orientation. ${ }^{28}$ From the plateau of $\varepsilon^{\prime}$ at $355 \mathrm{~K} \leq \mathrm{T} \leq 393 \mathrm{~K}$ (Fig. S1) the dielectric increment $\Delta \varepsilon_{\mathrm{T}}$ appears relatively small, with a value around unity at $\mathrm{T}_{\mathrm{g}}$, and as such it is compatible with the similarly small molecular dipole moment $\mu=1.34 \mathrm{D}$ calculated for the trifluoroethoxycarbonyl moiety of poly(TFEMA). ${ }^{29}$ The overall dynamic scenario for the different relaxations is shown in Fig. 3, also including a few data from literature for poly(TFEMA) $)^{30}$ and for the parent unfluorinated poly(EMA). ${ }^{31-33}$

While the two secondary processes, active in the glassy state have an Arrhenius behaviour, the relaxation times of the $\alpha$-relaxation above $T_{g}$ follow a non-Arrhenius law, with an apparent activation energy increasing with decreasing temperature, typical of structural processes in supercooled systems. $\alpha$-Relaxation can be fitted by the Vogel-Fulcher-Tamman (VFT) law:

$\tau(T)=\tau_{\infty} \exp \left[\frac{E_{V}}{R\left(T-T_{V}\right)}\right]$

where $\mathrm{R}$ is the gas constant, $\tau_{\infty}$ and $\mathrm{E}_{\mathrm{V}}$ are the relaxation time and the apparent activation energy at very high temperatures, respectively, and $T_{V}$ is the Vogel temperature, where ideally the relaxation time should diverge (for a simple Arrhenius behaviour, $\mathrm{T}_{\mathrm{V}}=0$ ). 


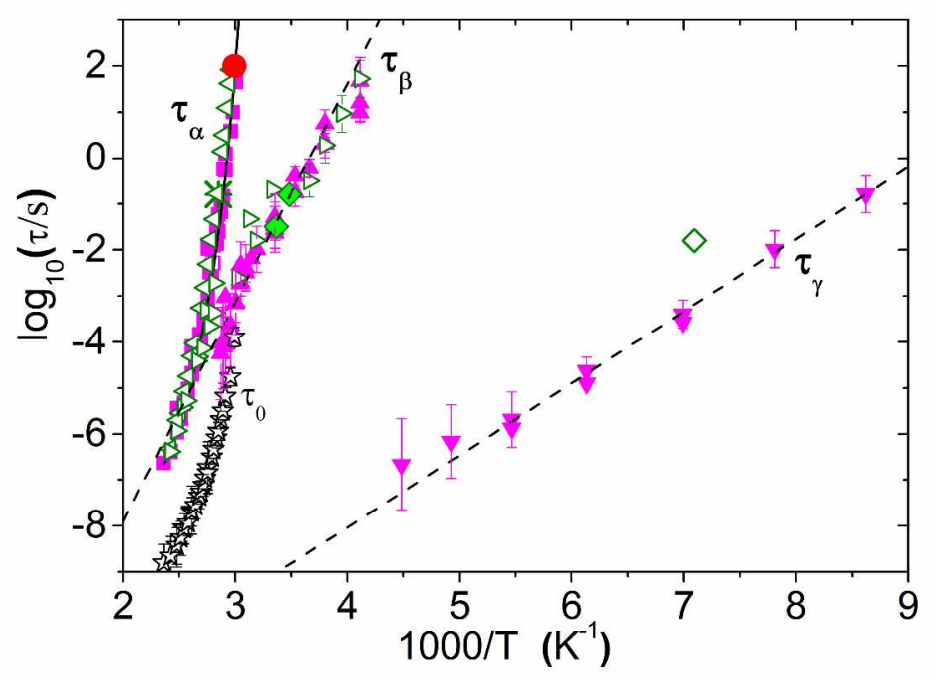

Figure 3. Relaxation map of poly(TFEMA): magenta squares, upward solid triangles and downward solid triangles represent $\tau_{\alpha}, \tau_{\beta}$ and $\tau_{\gamma}$, respectively. Green asterisk, solid diamonds and hollow diamond represent $\tau_{\alpha}, \tau_{\beta}$ and $\tau_{\gamma}$, respectively, from dynamical mechanical data from refs.[30, 34]. Solid red circle is from DSC (see text). The continuous line is a VFT fitting to $\tau_{\alpha}$; the dashed lines are for Arrhenius fits of secondary relaxation times. Open black stars show the primitive relaxation time, $\tau_{0}$, predicted by eq.(6). Green leftward and rightward hollow triangles represent $\tau_{\alpha}$ and $\tau_{\beta}$, respectively, of poly(EMA) [31, 32, 33], shown For comparison.

\begin{abstract}
A dielectric definition of $T_{g}$ is conventionally based on the temperature at which the $\alpha$ relaxation has $\tau_{\alpha}=100 \mathrm{~s}$. Similarly, a $\mathrm{T}_{\beta}$ and a $\mathrm{T}_{\gamma}$ transition temperature can also be defined for the secondary relaxations, corresponding to the temperature at which $\tau_{\beta, \gamma}=100 \mathrm{~s}$.

The $\alpha$-relaxation is usually ascribed to the structural process, and thus associated with the glass transition phenomenon, arising from the cooperative motions of several segments of the same or adjacent chains. The present dielectric spectroscopy data for the $\alpha$-relaxation (spanning over 9
\end{abstract}


decades from the glass transition) are in good agreement with our DSC results and with previous data from isochronal dynamic mechanical experiments ${ }^{34}$ as well as with those reported by Meskini et al., ${ }^{20}$ in which the explored dynamic range was, however, limited to 4 decades and far from the glass transition $(1 \mu \mathrm{s}<\tau<1 \mathrm{~ms})$.

A further comparison of $\alpha$-relaxation data for poly(TFEMA) with those of poly(EMA) reveals that both systems have a very similar dynamics, i.e. not only a very similar value for the glass transition temperature but also sharing the same temperature dependence, that can be estimated by the steepness index $\mathrm{m}$ (also termed "fragility") related to the apparent activation energy calculated at $\mathrm{T}_{\mathrm{g}}$, that is commonly associated to the degree of cooperativity of the relaxation:

$m=\left.\frac{\partial\left[\log _{10}\left(\tau_{\alpha}\right)\right]}{\partial\left(T_{g} / T\right)}\right|_{T_{g}}=\log _{10}(e) \frac{T_{g} E_{V}}{R\left(T_{g}-T_{V}\right)^{2}}$

The steepness index is $\mathrm{m}=90$ for the $\alpha$-relaxation of poly(TFEMA) and it is similar to that reported for the $\alpha$-relaxation of poly(EMA) $\left(\mathrm{m}=81, T_{g}=344 \mathrm{~K}\right) .{ }^{35}$ Apparently, the replacement of the methyl side chain end in poly(EMA) with the bulkier and more polar trifluoromethyl group in poly(TFEMA) has only a minor influence on the structural dynamics. In fact, the lower $\mathrm{T}_{\mathrm{g}}$ of poly(TFEMA) compensates for its higher fragility (directly related of cooperativity) with respect to poly(EMA), resulting in comparable dielectric relaxation times.

Concerning the secondary relaxation processes detected in the glassy state, the $\beta$-process shows an Arrhenius behaviour below $\mathrm{T}_{\mathrm{g}}$ whereas a deviation from this trend occurs above $\mathrm{T}_{\mathrm{g}}$. Such deviations of $\tau_{\beta}$ from the Arrhenius glassy behavior to shorter times have been clearly shown for several systems ${ }^{36}$ and attributed to the markedly increasing free volume on crossing the glass transition, affecting the intermolecular barrier that the $\beta$-process has to overcome. Nevertheless, the possibility of an artifact arising from the superposition fitting procedure cannot be excluded, as already pointed out by some authors. ${ }^{37}$ 
The $\beta$-relaxation time of glassy poly(TFEMA) is quite similar to those reported for poly(EMA) based on dynamic mechanical $^{25}$ and dielectric relaxation ${ }^{31,38}$ as well as NMR experiments. ${ }^{32}$ This $^{3}$ process is probably due to a $180^{\circ}$ flip rotation of the carboxy-ester side group coupled to a restricted rocking motion of a directly connected main chain segmental unit, as demonstrated by Spiess for PMMA and poly(EMA). ${ }^{32,33}$ According to this explanation, the $\beta$-relaxation partly requires the motions of the main chain and therefore shares the properties of the intermolecular Johari-Goldstein (JG) relaxation. ${ }^{39,40}$ This is also confirmed by overlapping of the experimental data above $\mathrm{T}_{\mathrm{g}}$ with those calculated according to the key equation of the Coupling Model (CM) (see Fig. 3):

$\tau_{\beta} \approx \tau_{0}=\tau_{\alpha}^{\beta_{K W W}} t_{c}^{1-\beta_{K W W}}$

where the time $t_{c}$ may be considered as a constant $\left(t_{c} \approx 2 p s\right)$ for molecular glass-formers and polymers, $\beta_{\mathrm{KWW}}$ is the stretching parameter of the Kohlrausch-Williams-Watt correlation function (see Supporting Information), $\tau_{0}$ is the primitive relaxation time calculated from the alpha relaxation parameters $\left(\tau_{\alpha}, \beta_{\mathrm{KWW}}\right)$ and $\tau_{\beta}$ is the experimental $\beta$-relaxation time, taken as the most probable value of the relaxation time distribution. ${ }^{41}$ An alternative way to check the validity of the coupling model is to look at the ratio between activation energy of the $\beta$-process in the glassy state and the glass transition temperature $\mathrm{T}_{\mathrm{g}}$. According to the $\mathrm{CM}^{42}$ this ratio is expressed as:

$$
E_{\beta} / R T_{g}=2.303\left(2-13.7 n-\log _{10} \tau_{\infty}\right)
$$

where $\mathrm{n}$ is the couping parameter and $\tau_{\infty}$ is the prefactor of the Arrhenius fit for the $\beta$ relaxation. Below $T_{g}$, the experimental value of this ratio, $E_{\beta(\exp )} / \mathrm{RT}_{\mathrm{g}} \sim 33 \pm 2$, and the $\mathrm{CM}$ calculated one, $\mathrm{E}_{\beta(\text { calc })} / \mathrm{RT}_{\mathrm{g}} \sim 31 \pm 2$, are actually in good agreement. 
The $\gamma$-relaxation process in glassy poly(TFEMA) shows activation energy, relaxation time and dielectric strength (see Fig. 3 and Tab. 1) comparable with those of a series of poly(fluoroalky1 methacrylate)s investigated by Koizumi and attributed to a local molecular motion of the perfluoroalkyl side chains. ${ }^{18}$ The relaxation map parameters are summarized in Tab. 1.

Table 1. Dielectric relaxation map and thermal parameters for poly(TFEMA)

\begin{tabular}{lllll}
\hline Relaxation process & $\begin{array}{l}\log _{10}\left(\tau_{\infty}\right) \\
{[\mathrm{s}]}\end{array}$ & $\begin{array}{l}\mathrm{E}_{\mathrm{V}} \\
{\left[\mathrm{kJ} \cdot \mathrm{mol}^{-1}\right]}\end{array}$ & $\begin{array}{l}\mathrm{T}_{\mathrm{V}} \\
{[\mathrm{K}]}\end{array}$ & $\begin{array}{l}\mathrm{T}_{\mathrm{x}}{ }^{(\mathrm{a})} \\
{[\mathrm{K}]}\end{array}$ \\
\hline$\alpha$ & $-11.1 \pm 0.3$ & $11.6 \pm 0.5$ & $287 \pm 1$ & $333 \pm 0.2^{(\mathrm{b})}$ \\
$\beta$ & $-17.4 \pm 1.0$ & $91 \pm 8$ & 0 & $245 \pm 5$ \\
$\gamma$ & $-14.3 \pm 1.0$ & $30 \pm 3$ & 0 & $96 \pm 5$
\end{tabular}

(a) Determined by dielectric spectroscopy as the temperature $T_{x}\left(x=\alpha_{1}, \alpha_{2}, \beta, \gamma\right)$ at which the respective relaxation time $\tau_{\mathrm{x}}$ is $10^{2} \mathrm{~s}$.

(b) The glass transition temperature, $\mathrm{T}_{\mathrm{g}}$, was also determined by DSC as $\mathrm{T}_{\mathrm{g} \text { (onset) }}=334 \mathrm{~K}$ (onset of the curve inflection from a heating scan at $10{ }^{\circ} \mathrm{C} / \mathrm{min}$ ) and $\mathrm{T}_{\mathrm{g} \text { (midpoint) }}=341$ (midpoint of the curve inflection).

\subsection{Dynamics of poly(VCN-co-TFEMA)}

Introduction of polar cyano groups as a result of copolymerization of TFEMA with VCN has dramatic effects on both the dynamic properties and on the absolute value of permittivity. Isochronal scans revealed the presence of several processes, as shown in the $\varepsilon^{\prime}$ and $\tan (\delta)$ plots of Fig. 4. In the region above glass transition temperature the molecular dipoles are able to orient under the external field. For the copolymer poly(VCN-co-TFEMA) both $\varepsilon^{\prime}$ and $\tan \delta$ are higher than in the case of the reference TFEMA homopolymer. At low frequencies, the real part of permittivity $\varepsilon^{\prime}$ presents two inflections associated to two relaxation processes, $\alpha_{1}$ and $\alpha_{2}$, as 
shown by arrows in Fig. 4a. Additionally, the d.c. conductivity contribution due to the drift of ionic impurities is responsible for the upturn of the low frequency isochronal $\tan (\delta)$ scan at high temperature (Fig. 4b). Only the faster of the two relaxation processes, $\alpha_{2}$, appears as a distinct peak while the slower $\alpha_{1}$ process is hidden by the d.c. conductivity contribution, $\sigma_{d c}$.

Below $\mathrm{T}_{\mathrm{g}}$ the dipolar orientation is mainly blocked, but a weak dependence of $\varepsilon^{\prime}$ on $\mathrm{T}$ or $\omega$ can still be found, due to the presence of two secondary $\beta$ - and $\gamma$-type relaxations. These relaxations contribute to the two successive humps of $\tan (\delta)$ on decreasing $T$ (Fig. 4b). Since the values for the dielectric strength $\Delta \varepsilon_{\beta}$ and $\Delta \varepsilon_{\gamma}$ of these relaxations are very low (less than 0.01 ), the real part of permittivity is almost constant and it is dominated by the high frequency permittivity $\varepsilon_{\infty} \sim 3$, a quite typical value for the atomic and electronic polarization (see Fig. 4a).

In Fig. 5a, selected representative isothermal spectra recorded for $\varepsilon^{\prime}$ in the temperature range from 363 to $433 \mathrm{~K}$ are shown. The electrode polarization is active in the lower frequency range and responsible for very high $\varepsilon^{\prime}$ values, a phenomenon clearly observable for the higher temperature isotherms, $403 \mathrm{~K}, 413 \mathrm{~K}, 433 \mathrm{~K}$. At higher frequencies the same isotherms present a plateau, followed by a drop of $\varepsilon^{\prime}$. While at $433 \mathrm{~K}$ the plateau is broad and centered at $2 \mathrm{kHz}$, well into the region free from the electrode polarization, at lower temperatures the same plateau shifts to lower frequencies and a second step of $\varepsilon^{\prime}$ with frequency is indicative of the presence of a second relaxation, $\alpha_{2}$, as discussed in the case of the reference homopolymer. Clearly, the polarization phenomenon shifts faster towards lower frequencies than the $\alpha$-relaxations. Below $373 \mathrm{~K}$ the $\alpha$-relaxations occur at frequencies too low to be observed. A more detailed picture can be obtained from a plot of $\varepsilon^{\prime \prime}(\omega, \mathrm{T})$ against the frequency, as shown in Fig. 5b. The contributions of the $\alpha_{1^{-}}, \alpha_{2^{-}}$, and $\beta$-relaxations, respectively, are indicated by the arrows. Both $\alpha_{1^{-}}$and $\alpha_{2^{-}}$ 
processes shift at lower frequencies on cooling but with different temperature dependence; as a consequence, they tend to merge on approaching the glass transition temperature.

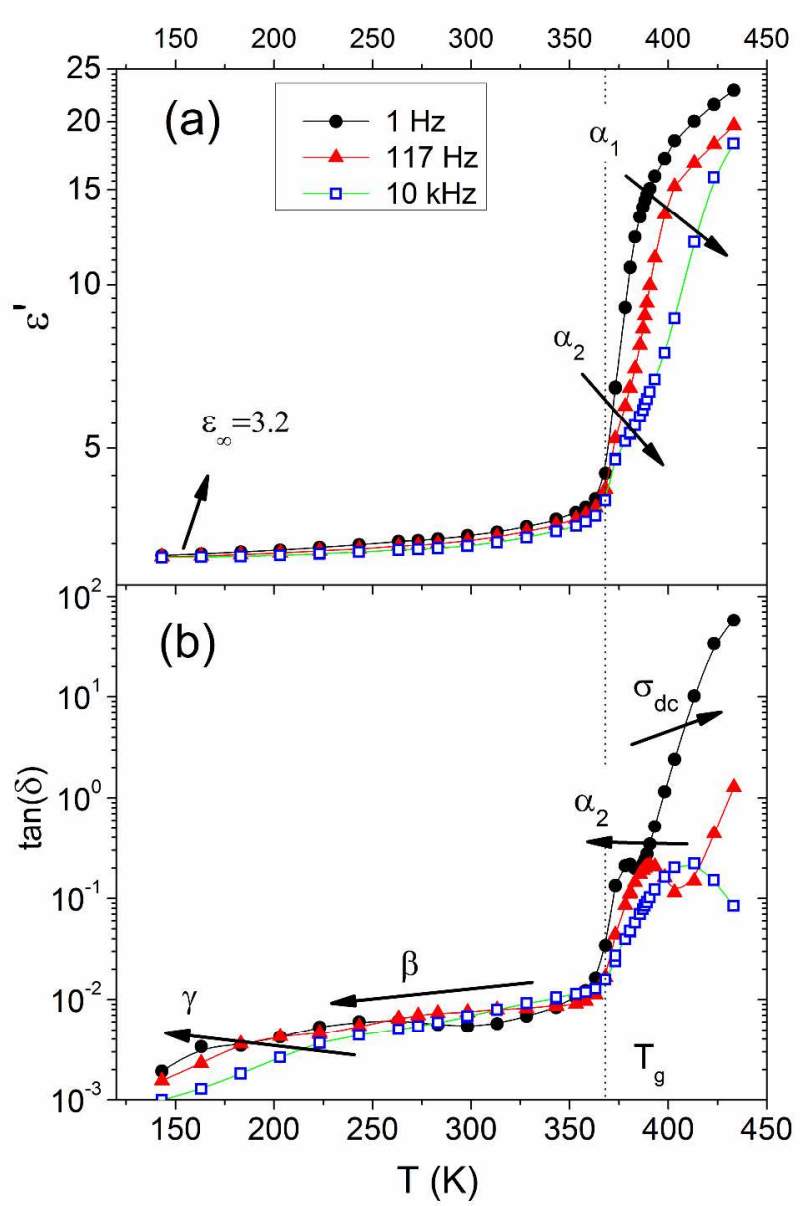

Figure 4. Poly(VCN-co-TFEMA) isochronal scans at $1 \mathrm{~Hz}, 117 \mathrm{~Hz}$ and $10 \mathrm{kHz}$ : a) dielectric permittivity, $\varepsilon^{\prime}$; b) loss tangent, tan $\delta$. Vertical dashed line indicates the location of the dielectric glass transition temperature (see Table 2). 


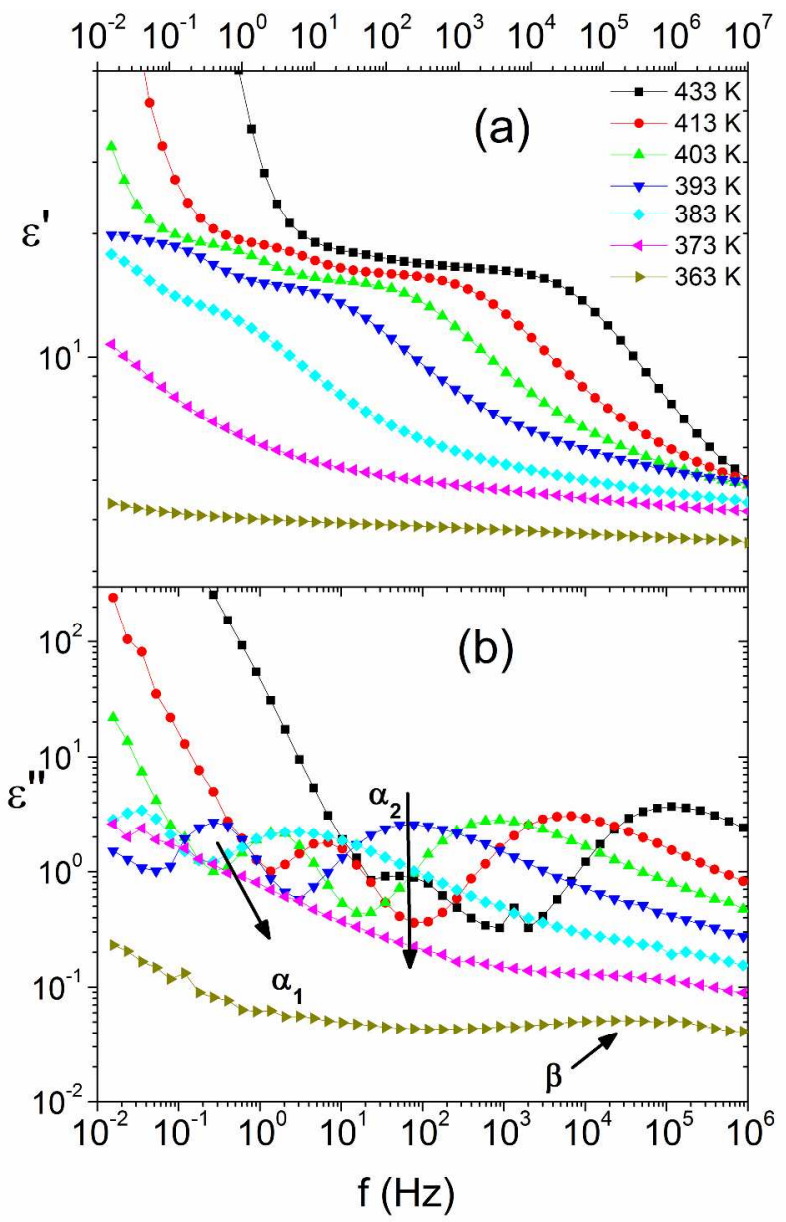

Figure 5. Selected isothermal spectra of poly(VCN-co-TFEMA) in the high temperature range (433-363 K): (a) permittivity ( $\left.\varepsilon^{\prime}\right)$; (b) dielectric loss ( $\left.\varepsilon^{\prime \prime}\right)$.

Following the previously discussed procedure, the contributions of the two relaxation processes, $\alpha_{1}$ - and $\alpha_{2^{-}}$, and that of d.c. conductivity can be deconvoluted by fitting eq.(1) to the spectra (see Fig. 6). 


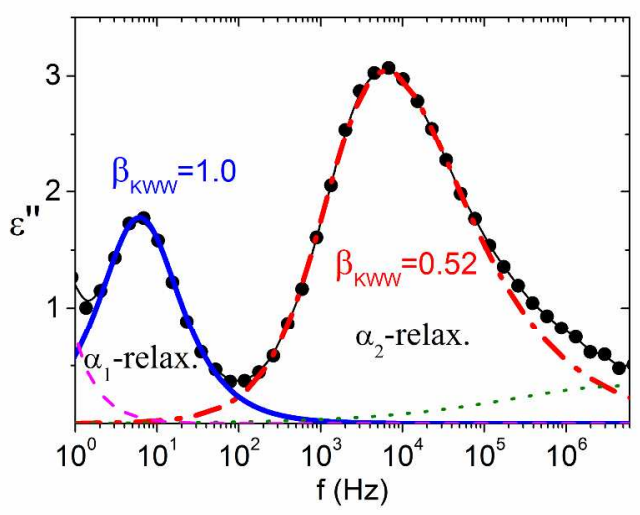

Figure 6. Representative fitting convolution curve (according to eq. 1, black thin solid line) of the isothermal loss spectrum (solid symbols) at $\mathrm{T}=413 \mathrm{~K}$ for poly(VCN-co-TFEMA), highlighting the individual contributions of $\alpha_{1}$ (blue thick solid line) and $\alpha_{2}$ (red thick dash-dot line) relaxations, as well as those of conductivity (magenta dashed line) and $\beta$-relaxation (green dotted line).

The $\beta$ - and $\gamma$ - secondary relaxations can be clearly detected from the dielectric loss spectra at lower temperatures (Fig. 7). It is apparent that the $\gamma$ - relaxation strength is only mildly affected by increasing temperature indicating that the related motion involve at a lesser extent the overall polarization fluctuation. On the other hand, the $\beta$-relaxation strength is strongly activated with temperature, thus becoming the dominant feature of the loss spectra above room temperature. 


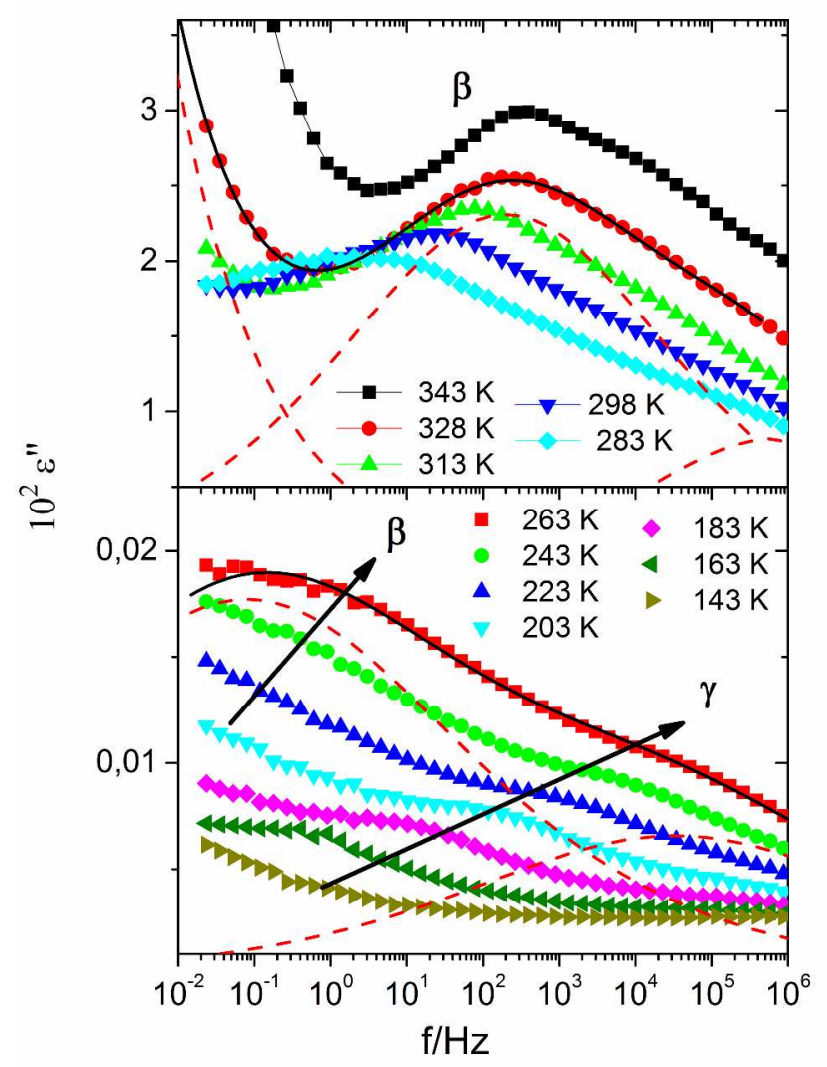

Figure 7. Selected isothermal dielectric loss spectra of poly(VCN-co-TFEMA) in the low temperature range (343-143 K). The contributions of the Havriliak Negami fitting functions of the spectra at $328 \mathrm{~K}$ (panel a) and $263 \mathrm{~K}$ (panel b) have been added for example. The total fitting curve is represented as continuous black line, the contributions are represented as red dashed lines. 


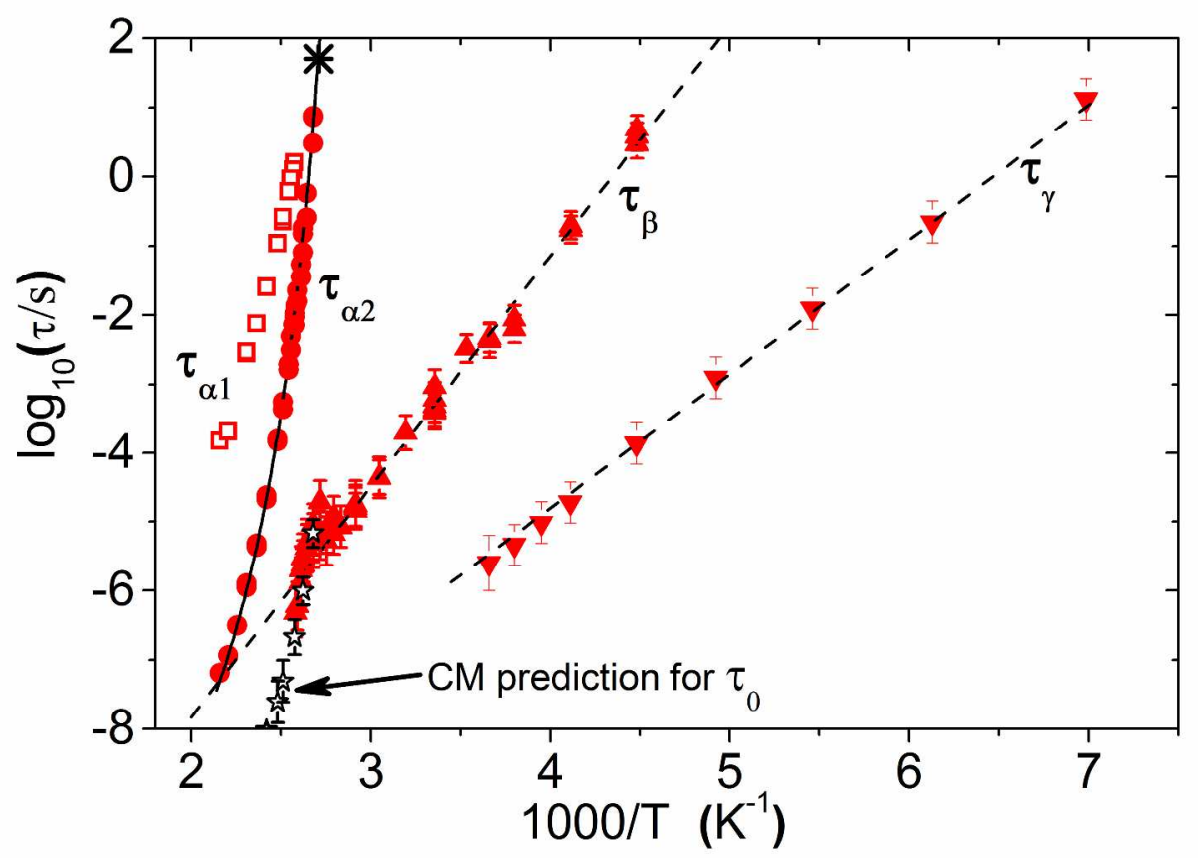

Figure 8. Relaxation map for poly(VCN-co-TFEMA). Red hollow squares and solid circles, upward triangles and downward triangles represent $\tau_{\alpha 1}, \tau_{\alpha 2}, \tau_{\beta}$ and $\tau_{\gamma}$ respectively. Black asterisk is from DSC (see text). The continuous line is a VFT fit to $\tau_{\alpha}$; the dashed lines are Arrhenius fits of secondary relaxation times. Hollow stars show the primitive relaxation time, $\tau_{0}$, from eq. (6).

An overall picture of the relaxation dynamics can be drawn from Fig. 8. The relaxation map shows that the characteristic times of the two secondary processes, $\beta$ - and $\gamma$-, have an Arrhenius behaviour, while the relaxation times of the two $\alpha$ processes follow the VFT law (eq. 4) with apparent activation energies increasing with decreasing temperature. In particular, the faster relaxation, $\alpha_{2^{-}}$, has a much stronger temperature dependence, its steepness indexes (eq. 5) being $\mathrm{m}\left(\alpha_{2}\right)=101 \pm 3$ and $\mathrm{m}\left(\alpha_{1}\right)=44 \pm 5$. As a consequence, the $\alpha_{1}$ - and $\alpha_{2}$ - relaxation times converge at $368 \mathrm{~K}$, corresponding to the calorimetric $\mathrm{T}_{\mathrm{g}}$ (onset). The complete list of fit parameters can be found in Tab. 2 . 
Table 2. Dielectric relaxation map and thermal parameters for poly(VCN-co-TFEMA)

\begin{tabular}{|l|l|l|l|l|}
\hline Relaxation process & $\log _{10}\left(\tau_{\infty} / \mathrm{s}\right)$ & $\begin{array}{l}\mathrm{E}_{\mathrm{V}} \\
{\left[\mathrm{kJ} \cdot \mathrm{mol}^{-1}\right]}\end{array}$ & $\begin{array}{l}\mathrm{T}_{\mathrm{V}} \\
{[\mathrm{K}]}\end{array}$ & $\begin{array}{l}\mathrm{T}_{\mathrm{x}} \\
{[\mathrm{K}]}\end{array}$ \\
\hline$\alpha_{1}$ & $-9.5 \pm 0.3$ & $21 \pm 2$ & $275 \pm 8$ & 371 \\
\hline$\alpha_{2}$ & $-12.5 \pm 0.1$ & $15 \pm 1$ & $315 \pm 2$ & $368^{(\mathrm{b})}$ \\
\hline$\beta$ & $-14.5 \pm 0.9$ & $64 \pm 5$ & 0 & 202 \\
\hline$\gamma$ & $-12.9 \pm 0.9$ & $39 \pm 3$ & 0 & 134 \\
\hline
\end{tabular}

(a) Determined by dielectric spectroscopy as the temperature $T_{x}\left(x=\alpha_{1}, \alpha_{2}, \beta, \gamma\right)$ at which the respective relaxation time $\tau_{\mathrm{x}}$ is $10^{2} \mathrm{~s}$.

(b) The glass transition temperature, $\mathrm{T}_{\mathrm{g}}$, was also determined by DSC as $\mathrm{T}_{\mathrm{g}(\mathrm{onset})}=368 \mathrm{~K}$ (onset of the curve inflection from a heating scan at $10{ }^{\circ} \mathrm{C} / \mathrm{min}$ ) and $\mathrm{T}_{\mathrm{g} \text { (midpoint) }}=373$ (midpoint of the curve inflection).

Both the glass transition temperature $\mathrm{T}_{\mathrm{g}}=373 \mathrm{~K}$ and the steepness index $\mathrm{m}=101$ of poly(VCNco-TFEMA) are quite higher than those of the reference TFEMA homopolymer $\left(\mathrm{T}_{\mathrm{g}}=341 \mathrm{~K}\right.$, $\mathrm{m}=90)$. Its $\alpha$-relaxation dynamics is much slower than that of both poly(TFEMA) and the parent TFEMA copolymers of similar composition with either methacrylonitrile (MAN) in poly(MANco-TFEMA) or acrylonitrile (AN) in poly(AN-co-TFEMA) ${ }^{19,20}$ Thus the presence of the cyano groups causes a progressive reduction of the conformational mobility of the main chain and a larger activation energy for the onset of the segmental motions at $\mathrm{T}_{\mathrm{g}}$. This is possibly a consequence of the different microstructure of poly(VCN-co-TFEMA), a more regularly alternating copolymer than those of TFEMA with either MAN or AN, both less electron-poor monomers than VCN. Such a higher microstructural uniformity and the consequent homogeneity 
of the local density of the strong $\mathrm{CN}$ dipoles is likely to increase the interaction between the chains, thus lowering the free volume.

It is noteworthy the difference of the relaxation shape of the two $\alpha$ processes, as exemplified in Fig. 6. Whereas the slowest process $\left(\alpha_{1^{-}}\right)$is a Debye-like peak, i.e. with the HN shape parameters a and $b$ of eq. 1 both equal to 1 , the $\alpha_{2}$ - loss peak has a broad and asymmetric shape, further broadening as the temperature is lowered: the calculated $\mathrm{HN}$ shape parameters were $a=0.85$ and $b=0.35$ at $403 \mathrm{~K}$, and $\mathrm{a}=0.68$ and $\mathrm{b}=0.38$ at $386 \mathrm{~K}$. An alternative description of the $\alpha_{2}$-relaxation shape, in terms of Kohlrausch-Williams-Watt correlation function, results in a stretching parameter value $\beta_{\mathrm{KWw}}$ of about 0.5 . Such broadness of the relaxation peak is typical of cooperative segmental processes close to the glass transition, experiencing a large dynamical heterogeneity. ${ }^{43}$ On the other hand, the narrow and temperature independent shape of the $\alpha_{1}$ process may be associated with slow motions occurring on a larger length scale and thus sensing an averaged dynamic environment, ${ }^{44,45}$ resulting in loss of information on the dynamic heterogeneity. While no previous report mentions the presence of cooperativity in either cyanoacrylic or VCN homo and copolymers, two cooperative relaxations have been detected and described in a series of liquid crystalline homopolymers with cyanobenzene side groups; in these systems the two cooperative relaxations involve to a different extent segmental motion $(\alpha-$ process) and mesogenic group reorientation, ${ }^{46}$. In this and several other examples (e.g. Xing K., et al. $^{47}$ ), the faster relaxation (with a shorter relaxation time) shows a stronger temperature dependence, i.e. a higher steepness index $\mathrm{m}$, than the slower relaxation. As a consequence, as the temperature decreases, the two relaxation times approach and so does the temperature at which the two dynamic processes become very long. 
The temperature dependence of the $\alpha_{1}$ - and $\alpha_{2}$ - relaxation times is in agreement with the above observations. In fact, while the merging of the relaxation times of the two processes around the calorimetric $\mathrm{T}_{\mathrm{g}}$ indicated a shared relation with the structural dynamics, the higher steepness of the $\alpha_{2}$-process points out that only this relaxation has a true cooperative character. Similar values of the $m$ index are typically observed for the cooperative motions of several segments of the same or adjacent chains in conformationally rigid polymers. ${ }^{35}$

As for the $\alpha_{1}$-process, its Debye-like loss peak with a quite small steepness $m$ index may be associated with dipole fluctuations occurring on a length scale larger than that typical of an $\alpha$ process, such as those reported for end-to-end or helical relaxations. ${ }^{45,48}$

A further evidence of the different microscopic nature of the two processes was obtained by comparing the results discussed so far with those from a poly(VCN-co-TFEMA) film obtained by using acetonitrile instead of chloroform as a casting solvent. In fact, all the parameters (relaxation times, shape factors, relaxation strength) drawn from the dielectric spectra are invariant, with the exception of a higher dielectric strength of $\alpha_{1}$-relaxation process measured for the film cast from acetonitrile solution, as shown from dielectric loss at $413 \mathrm{~K}$ in Fig. 9.

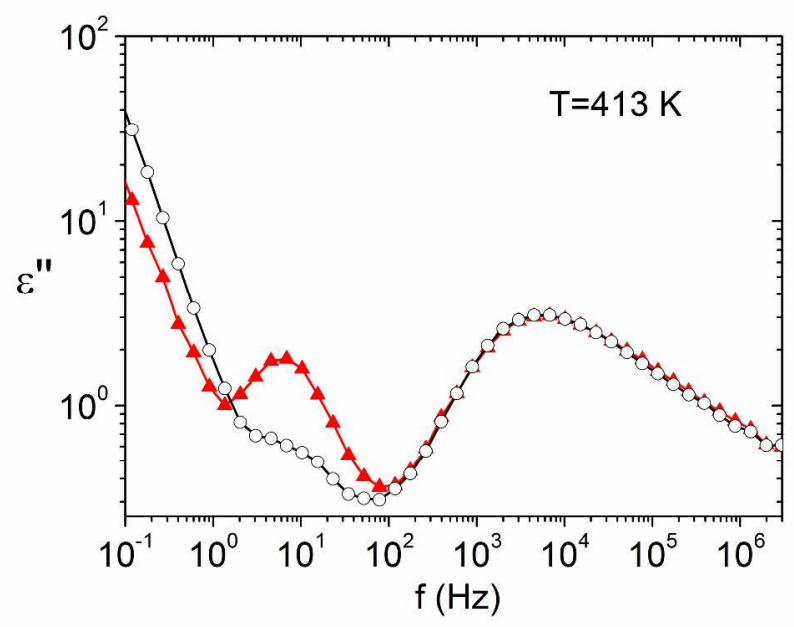


Figure 9. Dielectric loss of poly(VCN-co-TFEMA) film cast from solution in acetonitrile (solid triangles) and chloroform (open circles). Isothermal spectra recorded at $413 \mathrm{~K}$, after conditioning at $400 \mathrm{~K}$ in vacuum overnight.

Such effect persists at temperatures as high as $70 \mathrm{~K}$ above $T_{g}$, when the $\Delta \varepsilon$ of $\alpha_{1}$-relaxation reduces to lower values, comparable to those obtained for the film cast from chloroform. It is apparent that the highly polar acetonitrile solvent promotes the formation of locally aligned domains of dipoles, as discussed later. It is noteworthy that the application of a d.c. bias to the film, again at $413 \mathrm{~K}$, further magnifies the observed increase of the dielectric strength of $\alpha_{1^{-}}$ relaxation in the acetonitrile cast film (Fig. 10), an effect that is relatively rapid in its onset (faster than spectrum acquisition time, about ten minutes) but decays with a very slow kinetics once the bias is removed. As a possible explanation one may consider the better solvency of acetonitrile that is likely to act as plasticizer in the final stage of film formation, thus allowing cooperative conformational rearrangements resulting in the generation of local domains with aligned dipoles. Once generated, these domains are quite stable and can be further oriented upon the application of the bias.

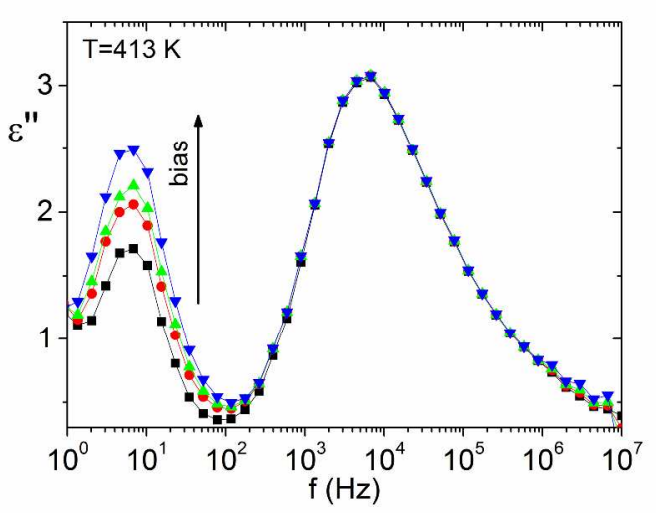

ACS Paragon Plus Environment 
Figure 10. Dielectric loss at $413 \mathrm{~K}$ of poly(VCN-co-TFEMA) film cast from solution in acetonitrile measured with increasing applied d.c. bias voltage: $0 \mathrm{~V}$ (squares), $100 \mathrm{~V}$ (circles), $200 \mathrm{~V}$ (upward triangles), $400 \mathrm{~V}$ (downward triangles).

Differently from the observations on the the $\alpha_{1}$ - and $\alpha_{2}$ - relaxations, the cyano groups do not affect significantly the nature of the low temperature $\beta$ - and $\gamma$-relaxations, both characterized by low dielectric strengths. By analogy with our results on the reference poly(TFEMA) and with previous investigations on poly(fluoroalkyl $\alpha$-substituted acrylate)s, ${ }^{49}$ the $\beta$ - and $\gamma$-relaxations are attributed to a rotational motion of carboxy ester groups in the glassy region also involving restricted motions of the main chain, ${ }^{32}$ and an internal motion of fluoroalkyl side chains, respectively. In fact, dipolar fluctuations associated with conformational motions involving cyano groups are negligible in the glassy state, while those associated with vibrational modes are not relevant since they take place over a much faster timescale, as already demonstrated by previous studies on VCN-vinyl ester copolymers. ${ }^{50,51}$ One may wonder why a low activation energy of $\beta$-relaxation $(64 \mathrm{~kJ} / \mathrm{mol}$ in the copolymer compared with $91 \mathrm{~kJ} / \mathrm{mol}$ in the reference homopolymer) is associated with a higher $T_{g}$ and steepness index m (Tab. 2). The higher $T_{g}$, and thus a lower bulk free volume, can be attributed to stronger interchain interactions associated with presence of the highly polar cyano groups. On the other hand, in the nearly alternating poly(VCN-co-TFEMA) the carboxy ester group, responsible for $\beta$ relaxation, is generally vicinal to a much less bulky VCN unit, thus experiencing lower steric hindrance and rotational energy barrier. The introduction of VCN unit in the copolymer has thus the effect of separating the $\alpha$ and $\beta$ - relaxation timescales. 
It is noteworthy that, applying the CM prediction (eq. 6) to the case of the copolymer, the primitive relaxation $\tau_{0}$ is in good agreement with experimental $\beta$-relaxation time. The higher separation between $\alpha$ - and $\beta$ - relaxation time is thus related to the smaller $\beta_{\mathrm{kww}}(\sim 0.5)$ measured for the copolymer, explained by the enhanced cooperativity and rigidity of macromolecules. A similar situation concerning the $\alpha-\beta$ separation as a result of the introduction of rigid comonomer in polymers of dielectrically active monomer unit has been reported by Kahle ${ }^{52}$ and accordingly discussed in the framework of Coupling Model. ${ }^{53}$

The relative contributions of the $\alpha_{1}$ - and $\alpha_{2}$ - relaxation to the overall dielectric strength $\Delta \varepsilon_{\mathrm{T}}$ above $\mathrm{Tg}$ and their temperature dependence can give important information about the dipolar arrangement. As it can be seen in Fig. 11, the $\Delta \varepsilon_{\mathrm{T}}$ value for the copolymer is much higher than that of the reference homopolymer, as expected by the introduction of the very strong dipole moment associated to $\mathrm{CN}$ group. However, despite the different chain rigidity, the two systems (homo- and co-polymer) exhibit a similar temperature dependence of $\Delta \varepsilon_{\mathrm{T}}$, i.e. a first order polynomial of reciprocal temperature (see Fig. 11). Nevertheless, by considering the values of the Kirkwood factor $\mathrm{g}_{\mathrm{K}}$ (eq. 3) shown in the inset of Fig. 11, related to intra- and inter-molecular contributions to the ordering of dipoles, a striking difference is observed between the slight decrease with the temperature for the homopolymer, typical of molecular correlations from cooperative motions, and the nearly temperature-independent $\mathrm{g}_{\mathrm{K}}$ for the copolymer, in agreement with the local ordering imposed by the strong dipolar interactions among the VCN cyano groups. 


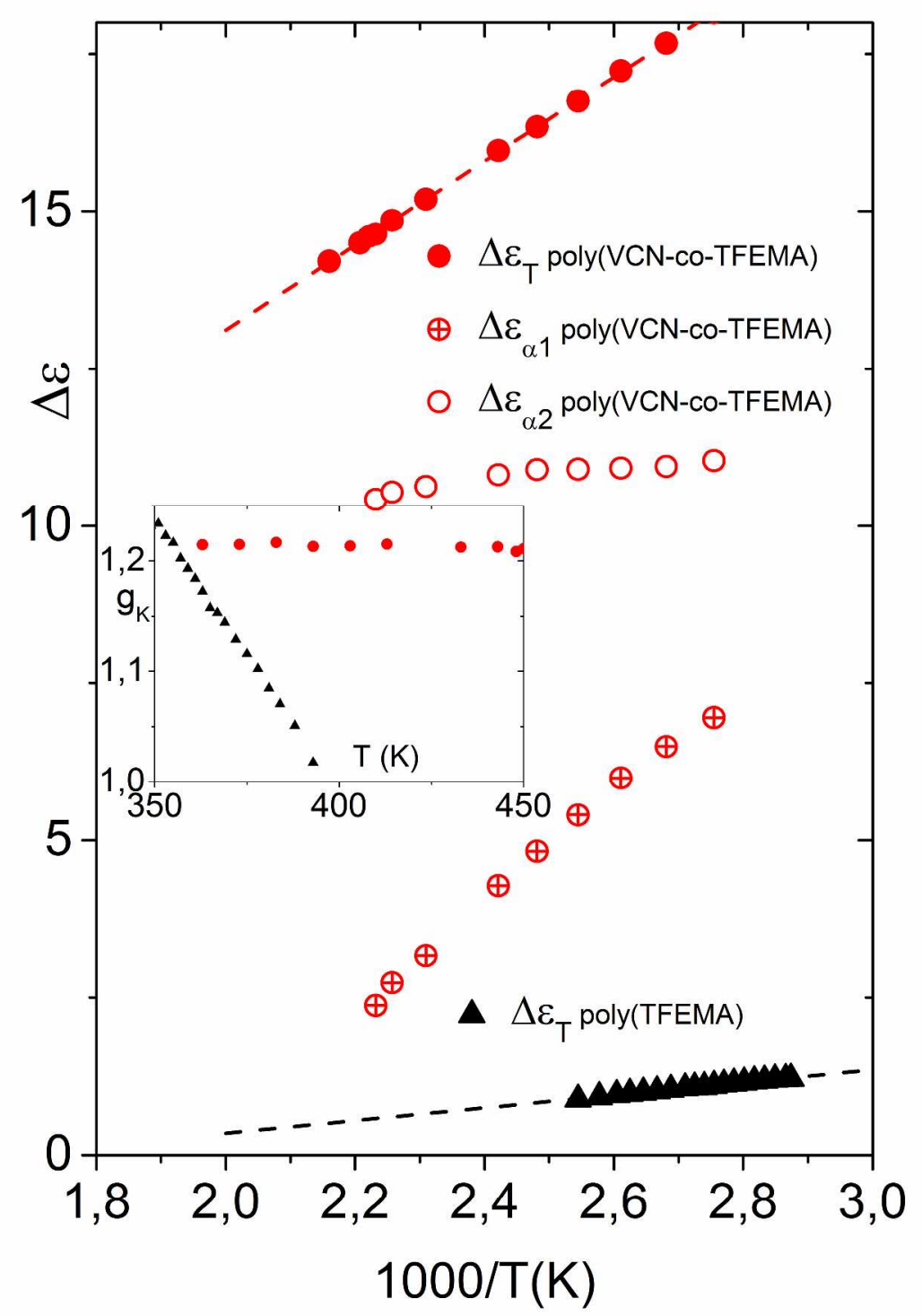

Figure 11. Overall dielectric strength $\Delta \varepsilon_{\mathrm{T}}$ of poly(VCN-co-TFEMA) (red solid circles) and poly(TFEMA) (black solid triangles) plotted as a function of reciprocal temperature (dashed lines are linear regressions to data). In addition, the dielectric strength of both $\alpha_{1^{-}}$and $\alpha_{2^{-}}$ processes (red crossed and open circles, respectively) of poly(VCN-co-TFEMA) as a function of reciprocal temperature is also shown. Inset shows the Kirkwood factor $g_{K}$ (eq. 3) for poly(VCNco-TFEMA) and poly(TFEMA) (red circles and black triangles, respectively) as a function of temperature. 
More interestingly, the relative contributions of the two $\alpha$-processes to the copolymer dielectric strength show different temperature behaviour. The dielectric strength of the slower process $\left(\alpha_{1^{-}}\right)$increases with decreasing temperature much faster than that associated with the faster process $\left(\alpha_{2^{-}}\right)$. As a highlight, in the temperature range between 403 and $383 \mathrm{~K}$ the value of $\Delta \varepsilon_{\alpha 1}$ increases from 4.82 to 6.0 , while $\Delta \varepsilon_{\alpha 2}$ remains practically unchanged from 10.90 to 10.92 . By extrapolation, at $\mathrm{T}_{\mathrm{g}}(361 \mathrm{~K})$ one obtains $\Delta \varepsilon_{\alpha 1}=7.15$ and $\Delta \varepsilon_{\alpha 2}=11.1$. Both values are close to that found for the single $\alpha$-process of similar cyano-functional polymers such as poly(VCN-cochlorostyrene $)(\Delta \varepsilon=13.1),{ }^{12}$ poly(MVCN-co-fluorostyrene $)(\Delta \varepsilon=10.9)$ and poly(MVCN-cochlorostyrene) $(\Delta \varepsilon=10.1)$, all of them being regularly alternating copolymers. ${ }^{7}$ Actually, if the sum of the relaxation strengths of both $\alpha_{1-}$ and $\alpha_{2^{-}}$processes in poly(VCN-co-TFEMA) is considered, then $\Delta \varepsilon_{\mathrm{T}}=17.2$ at $383 \mathrm{~K}$. Such a high value is similar to that of another alternating fluorinated $\mathrm{VCN}$ copolymer, poly(VCN-co-fluorostyrene) $(\Delta \varepsilon=23.4){ }^{7}$ and to that of various cyanovinyl acetate (CVA) polymers characterized by a different (a single cyano group every repeat unit) but again uniform distribution of cyano side groups along the macromolecular chain, such as poly(CVA) ( $\Delta \varepsilon=18.0)$, poly(AN-co-CVA) $(\Delta \varepsilon=14.8)$ and poly(MAN-co-CVA) ( $\Delta \varepsilon=24.4$ ) (the latter two being statistic copolymers). ${ }^{54}$ On the other hand, much lower values of $\Delta \varepsilon=8$ and $\Delta \varepsilon=11$ have been reported for TFEMA copolymers with less uniform distribution of cyano side groups, namely poly(AN-co-TFEMA) and poly(MAN-co-TFEMA), respectively. ${ }^{20}$

It is worth recalling here that the piezoelectric response of a material is related to the residual polarization $\mathrm{P}_{\mathrm{r}}=\Delta \varepsilon \cdot \varepsilon_{0} \cdot \mathrm{E}_{\mathrm{p}}$ achieved upon electric poling and after relaxation of the electronic and atomic polarizations at room temperature once the field $\mathrm{E}_{\mathrm{p}}$ is removed. ${ }^{55}$ Measuring the dielectric 
relaxation strength, $\Delta \varepsilon$, is therefore a practical way to evaluate the potential piezoelectricity of an amorphous polymer. On the other hand, in order to possess a large dielectric relaxation strength and hence piezoelectric response an amorphous polymer must feature a high concentration of highly polar groups and cooperative dipole motion.

The 3D arrangement associated with the two types of repeat units in the VCN-TFEMA-VCN trimer has been obtained by DFT simulation (see Fig. 12). The moduli of the dipole moments have been reported to be between 4.0 and 4.5 D for the highly polar VCN repeat unit ${ }^{56}$ and 1.34 $\mathrm{D}^{29}$ for the trifluoroethoxycarbonyl side chain of the TFEMA repeat unit.

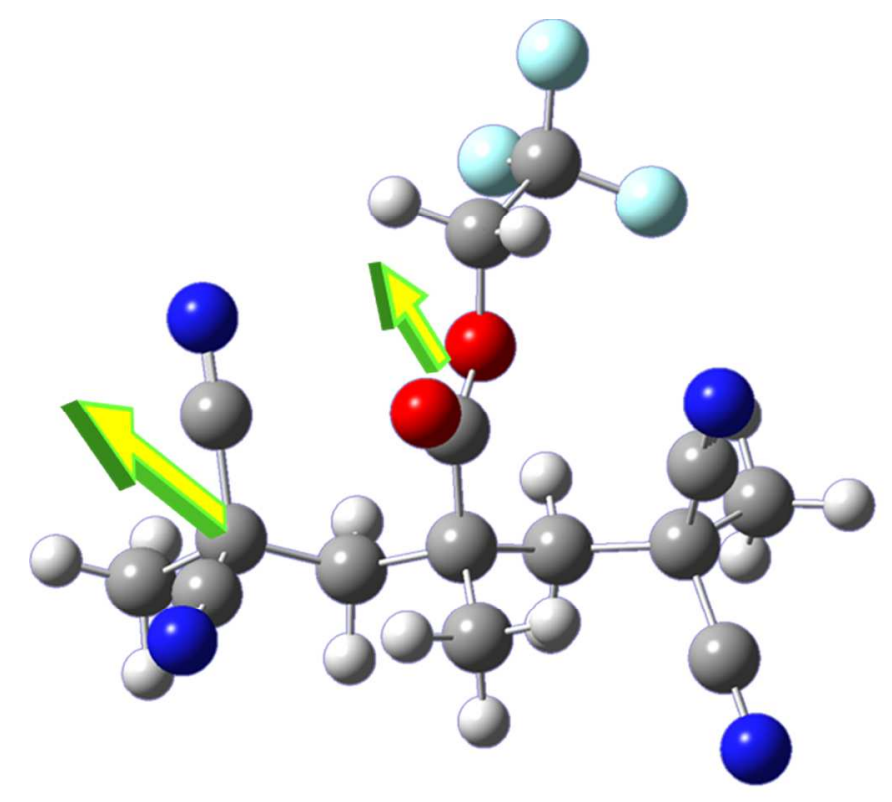

Figure 12. 3D molecular structure of a typical alternating poly(VCN-co-TFEMA) trimer. Energy minimized conformation showing the alignment of dipoles. The green arrows indicate the dipoles of the VCN unit and the trifluoroethoxycarbonyl side chain moiety of the TFEMA unit, respectively. 
The presence of a second $\alpha$-type relaxation may play an important role in the onset of such a high $\Delta \varepsilon$ value. The unusual finding of a second $\alpha$-process, may be explained with the structural features of the copolymer, characterized by a relatively high fraction of highly polar, alternating VCN-TFEMA dyads and, in particular, with a large fraction (about $90 \%$ according to a previous microstructural investigation) ${ }^{22}$ of TFEMA units with at least one vicinal VCN co-unit.

The likely occurrence of homosequences of alternating dyads long enough to involve independently activated segmental motions (about 5 dyads in nearly planar zigzag main chain conformation) corresponds to the structural length of about $2 \mathrm{~nm}$. This is generally considered as the lower length scale to allow time-scale separation from the $\alpha_{2}$-type relaxation, that is related to the segmental relaxation threshold for such relaxation to occur), and the energy minimized conformation of the alternating VCN-TFEMA-VCN triad shown in Fig. 12 shows the respective dipoles as nearly perfectly aligned, suggesting that under suitable conditions distinct microdomains may exist as a result of the cooperative orientation of the dipoles accounting for the observed additional $\alpha_{1}$-type relaxation.

\section{Conclusions}

A complex relaxation pattern was observed for poly(VCN-co-TFEMA) involving four processes: two below $T_{g}(\beta$ - and $\gamma-)$ and two distinct ones above $T_{g}\left(\alpha_{1^{-}}\right.$and $\left.\alpha_{2}-\right)$ but merging at low temperatures. Compared to a reference TFEMA homopolymer, the introduction of polar VCN units results in an increase of both dielectric constant and glass transition temperature. In addition, the cooperativity character of the structural relaxation is increased in poly( $\mathrm{VCN}-\mathrm{co}$ TFEMA), as shown by the larger fragility index, the broadness of the $\alpha_{2}$-relaxation peak, and the larger separation between the $\alpha$ - and $\beta$-relaxation timescales. 
Such cooperativity has been already discussed in a previous work by Tasaka et al. ${ }^{57}$ concerning strictly alternating copolymers of $\mathrm{VCN}$ with vinyl esters, and ascribed to the occurrence of strong dipolar interactions leading to local aggregation and weak micro-ordering involving chain segments, that could only be destroyed well above $\mathrm{T}_{\mathrm{g}}$. The very high $\mathrm{T}_{\mathrm{g}}$ and dielectric strength observed by Tasaka had been attributed to the relatively rigid main chain and bulky side chain of these copolymers of VCN with vinyl esters, leading to a mesophase-like organization, in which only cooperative intermolecular motions are permitted. This ordering would contribute to the stabilization of the residual polarization upon poling, resulting in a piezoelectricity markedly stable below $\mathrm{T}_{\mathrm{g}}$ even in the absence of a crystalline phase.

In the case of poly(VCN-co-TFEMA) a similar behavior is observed, although the relaxation pattern does not lead to such an extended cooperative reorientation even upon poling at $\mathrm{T}=\left(\mathrm{T}_{\mathrm{g}}+40 \mathrm{~K}\right)$, possibly because of the higher conformational stiffness of the methacrylic TFEMA co-units with respect to the vinyl ester ones. The apparent contradiction of a less flexible methacrylic comonomer unit which, although less flexible than the vinyl ester one, leads to a lower $\mathrm{T}_{\mathrm{g}}$ of the respective $\mathrm{VCN}$ copolymer, may be explained with a less efficient conformational rearrangement that partially hinders the onset of an effective cooperative interaction.

The presence of rigid VCN co-units uniformly distributed in a nearly alternated comonomer sequence was shown to provide the fluorinated TFEMA copolymer with one of the highest values of dielectric strength for polymers around $\mathrm{T}_{\mathrm{g}}$, and to induce a cooperativity that plays a key role in the stabilization of oriented domains after electrical poling. The behavior of this type of copolymers broadens the range of amorphous soft materials with potential piezo-and pyroelectric properties. 


\section{Supporting Information}

Details on dielectric spectra of poly(TFEMA) are provided. This material is available free of charge via the Internet at http://pubs.acs.org.

\section{AUTHOR INFORMATION}

\section{Corresponding Author}

* Valter Castelvetro. Email: valter.castelvetro@unipi.it

\section{Author Contributions}

The manuscript was written through contributions of all authors. All authors have given approval to the final version of the manuscript. \$These authors contributed equally. (match statement to author names with a symbol)

\section{Funding Sources}

Financial support for cooperative research came from the University of Pisa (International Academic Cooperation Support Action Program - Cooperation with the University Cadi Ayyad of Marrakech 2009-2012) and the Italian MIUR Cooperlink projet No. CII118NLTZ.

\section{ACKNOWLEDGMENT}

The DFT calculation was performed by Dr. Chiara Cappelli, from the Department of Chemistry and Industrial Chemistry of the University of Pisa. 


\section{REFERENCES}

(1) Gerhard-Multhaupt, R. Poly(vinylidene fluoride): a piezo-, pyro- and ferroelectric polymer and its poling behaviour. Ferroelectrics 1987, 75, 385-392.

(2) Kawai, H. Piezoelectricity of poly(vinylidene fluoride). Jpn. J. Appl. Phys. 1969, 8, 975976.

(3) Seilers, R. Modern Fluoropolymers; Scheirs, J. Ed.; Wiley: New York, 1997; p 487.

(4) Furukawa T. Piezoelectricity in Polymers. IEEE Trans. Electr. Insul. 1989, 24, 375-393.

(5) Miyata, S.; Yoshikawa, M.; Tasaka, S.; Ko, M. Piezoelectricity Revealed in the Copolymer of Vinylidene Cyanide and Vinyl Acetate. Polymer J. 1980, 12, 857-860.

(6) Furukawa, T.; Date, M.; Nakajima, K.; Kosaka, T.; Seo, I. Large Dielectric Relaxations in an Alternate Copolymer of Vinylidene Cyanide and Vinyl Acetate. Jpn. J. Appl. Phys. 1986, 25, $1178-1182$.

(7) Raihane, M.; Montheard, J. P.; Boiteux, G. Etude diélectrique de deux copolymères du méthylcyanure de vinylidène avec le 4-fluorostyrène et le 4-chlorostyrène. Macromol. Chem. Phys. 2000, 201, 2365-2370.

(8) Kishimoto, M.; Nakajima, K.; Seo, I. European Patent EP0264240 (A2) 1988, Mitsubishi Petrochemical Co. Ltd., Chem. Abstr., 109 (1988) 171102h.

(9) Way, T. F.; Hall, H. K. Jr. Novel copolymerizations of methyl vinylidene cyanide with electron-rich vinyl monomers. Polym. Bull. 1990, 24, 151-156. 
(10) Hall, H. K. Jr.; Padias, A. B.; Chu, G.; Lee, H.-Y.; Kalinin, I.; Sansone, M.; Breckenridge, G. Novel Cyano-Containing Copolymers of Vinyl Esters for Piezoelectric Materials. J. Polym. Sci., Part A: Polym. Chem. 1992, 30, 2341-2347.

(11) Montheard, J. P.; Boinon, B.; Raihane, M.; Pham, Q. T. Copolymerization of methylvinylidene cyanide with vinyl acetate: proton and carbon-13 NMR studies of the microstructure. Polym. Commun. 1991, 32, 567-569.

(12) Belfkira, A.; Sadel, A.; Montheard, J. P.; Boiteux, G.; Lucas, J. M.; Seytre, G. Dielectric studies of amorphous vinylidene cyanide alternating copolymers. Polymer, 1993, 34, 4015-4019.

(13) Inoue, Y.; Kawaguchi, K.; Maruyama, Y.; Yo, Y. S.; Chujo, R.; Seo, I.; Kishimoto, M. ${ }^{13} \mathrm{C}$ n.m.r. studies on the microstructure of piezoelectric copolymers of vinylidene cyanide. Polymer 1989, 30, 698-704.

(14) Ohta, Y.; Inoue, Y.; Chujo, R.; Kishimoto, M.; Seo, I. Microstructure of vinylidene cyanide copolymers with linear-chain fatty acid vinyl esters. Polymer 1990, 31, 1581-1588.

(15) Atlas, S.; Raihane, M.; Kharas, G. B.; Hendrickson, P. G.; Kaddami, H.; Arous, M.; Kallel, A. Novel Copolymers of Difluoro Ring-substituted 2-Phenyl-1,1-dicyanoethylenes with 4-Fluorostyrene: Synthesis, Structure and Dielectric Study. J. Macromol. Sci.: Part A 2012, 49, 1-14.

(16) Raihane, M.; Zerroukhi, A.; Kaddami, H.; Lahcini, M.; Boiteux, G. Dielectric properties of copolymers based on cyano monomers and methyl $\alpha$-acetoxyacrylate. Polym. Internat. 2013, $62,684-692$. 
(17) Maruyama, Y.; Yo, Y. S.; Inoue, Y.; Chujo, R.; Tasaka, S.; Miyata, S. Carbon-13 nuclear magnetic resonance studies on the microstructure of the copolymer of vinylidene cyanide and methyl methacrylate. Poymer 1987, 28, 1087-1092.

(18) Koizumi, S.; Tadano, K.; Tanaka, Y.; Shimidzu, T.; Kutsumizu, S.; Yano, S. Dielectric Relaxations of Poly(fluoroalkyl methacrylate)s and Poly(fluoroalky1 a-fluoroacrylate)s. Macromolecules 1992, 25, 6563-6567.

(19) Raihane, M.; Ameduri, B. Radical copolymerization of 2,2,2-trifluoroethyl methacrylate with cyano compounds for dielectric materials: Synthesis and characterization. J. Fluorine Chem. 2006, 127, 391-399.

(20) Meskini, A.; Raihane, M.; Ameduri, B.; Hakme, C.; Sage, D.; Stevenson, I.; Boiteux, G.; Seytre, G.; Kaddami, H. Dielectric behaviour of copolymers based on 2,2,2-trifluoroethyl methacrylate and cyano co-monomers. Eur. Polym. J. 2009, 45, 804-812.

(21) Merino, E. G.; Raihane, M.; Atlas, S.; Belfkira, A.; Lahcini, M. ; Hult, A. ; Dionísio, M. ; Correia, N. Molecular dynamics of poly(ATRIF) homopolymer and poly(AN-co-ATRIF) copolymer investigated by dielectric relaxation spectroscopy. Eur. Polym. J. 2011, 47, 14291446.

(22) Raihane, M.; Castelvetro, V.; Bianchi, S.; Atlas, S.; Ameduri. B. Radical Copolymerization of Vinylidene Cyanide with 2,2,2-Trifluoroethyl Methacrylate: Structure and Characterization. J. Polym. Sci.: Part A: Polym. Chem. 2010, 48, 4900-4908. 
(23) Castelvetro, V.; Raihane, M.; Bianchi, S.; Atlas, S.; Bonaduce, I. Thermal degradation behaviour of a nearly alternating copolymer of vinylidene cyanide with 2,2,2-trifluoroethyl methacrylate. Polym. Degrad. Stab. 2011, 96, 204-211.

(24) Ngai, K. L. Relation between some secondary relaxations and the $\alpha$ relaxations in glassforming materials according to the coupling model. J. Chem. Phys. 1998, 109, 6982-6994.

(25) McCrum, N. G.; Read B. E.; Williams, G. Anelastic and dielectric effects in polymeric solids; Wiley: New York, 1991.

(26) Havriliak, S.; Negami, S. A complex plane representation of dielectric and mechanical relaxation processes in some polymers. Polymer 1967, 8, 161-210.

(27) Kremer, F.; Schönhals, A. (eds) Broadband Dielectric Spectroscopy; Springer-Verlag: Berlin, 2003.

(28) Böttcher, C. J. F.; Bordewijk, P. Theory of Electric Polarization, 2nd ed.; Elsevier: Amsterdam, 1978; Vol. 2.

(29) Raihane, M.; Ameduri, B. Fluoropolymer Dielectrics, in: Handbook of Fluoropolymer Science and Technology; Smith, D. W., Iacono S. T., Iyer S. S., Eds.; Wiley : New York, 2014; p 451-494.

(30) Higgenbotham-Bertolucci, P. R.; Harmon, J. P. Photonic \& Optoelectronic Polymers; ACS Symp. Ser.: 1997; p. 79-97.

(31) Ishida, Y.; Yamafuji, K. Studies on dielectric behaviors in a series of polyalkylmethacrylates. Kolloid-Z. 1961, 177, 97-116. 
(32) Kuebler, S. C.; Schaefer, D. J.; Boeffel, C.; Pawelzik, U.; Spiess, H. W. 2D Exchange NMR Investigation of the $\alpha$-Relaxation in Poly(ethyl methacrylate) as Compared to Poly(methyl methacrylate). Macromolecules 1997, 30, 6597-6609.

(33) Wind, M; Graf, R; Heuer, A; Spiess, H. W. Structural Relaxation of Polymers at the Glass Transition: Conformational Memory in Poly(n-alkylmethacrylates). Phys. Rev. Lett. 2003, 91, $155702-1 / 4$.

(34) Higgenbotham-Bertolucci, P. R.; Gao, H.; Harmon, J. P. Creep and Stress Relaxation in Methacrylate Polymers: Two Mechanisms of Relaxation Behavior Across the Glass Transition Region. Polym. Eng. Sci. 2001, 41, 873-880.

(35) Qin, Q.; McKenna, G.B. Correlation between dynamic fragility and glass transition temperature for different classes of glass forming liquids. J. Non-Cryst. Solids 2006, 352, 29772985.

(36) Kessairi, K.; Capaccioli, S.; Prevosto, D.; Lucchesi, M.; Sharifi, S.; Rolla, P. A. Interdependence of Primary and Johari-Goldstein Secondary Relaxations in Glass-Forming Systems. J. Phys. Chem. B 2008, 112, 4470-4473.

(37) Bergman, R.; Alvarez, F.; Alegría, A.; Colmenero, J. The merging of the dielectric $\alpha$ - and $\beta$-relaxations in poly-(methyl methacrylate). J. Chem. Phys. 1998, 109, 7546-7555.

(38) Schroter, K.; Unger, R.; Reissig, S.; Garwe, F.; Kahle, S.; Beiner, M.; Donth E. Dielectric Spectroscopy in the Râ Splitting Region of Glass Transition in Poly(ethyl methacrylate) and Poly(n-butyl methacrylate): Different Evaluation Methods and Experimental Conditions. Macromolecules 1998, 31, 8966-8972. 
(39) Johari, G. P.; Goldstein, M. Viscous Liquids and the Glass Transition. II. Secondary Relaxations in Glasses of Rigid Molecules. J. Chem. Phys. 1970, 53, 2372-2388.

(40) Capaccioli, S.; Paluch, M.; Prevosto, D.; Wang, L.-M.; Ngai, K. L. Many-Body Nature of Relaxation Processes in Glass-Forming Systems. J. Phys. Chem. Lett. 2012, 3, 735.

(41) Ngai, K. L. Relaxation and Diffusion in Complex Systems; Springer: New York, 2011.

(42) Ngai, K. L.; Capaccioli, S. Relation between the activation energy of the Johari-Goldstein $\beta$ relaxation and Tg of glass formers. Phys. Rev. E 2004, 69, 031501.

(43) Ediger, M. D. Spatially heterogeneous dynamics in supercooled liquids. Annu. Rev. Phys. Chem. 2000, 51, 99-128.

(44) Huang, W.; Richert, R. From heterogeneous probe rotation to the hydrodynamic limit. $J$. Non-Cryst. Solids 2006, 352, 4704-4709.

(45) Gainaru, C.; Hiller, W.; Böhmer, R. A Dielectric Study of Oligo- and Poly(propylene glycol). Macromolecules 2010, 43, 1907-1914.

(46) Nikonorova, N.; Pissis, P. Molecular Mobility in Liquid Crystalline Side-Chain Polyacrylates and Polymethacrylates with Cyanoazobenzene Side Groups: Dielectric Spectroscopy and Thermally Stimulated Depolarization Currents. Mol. Cryst. Liq. . 2015 623, 424-432.

(47) Xing, K.; Chatterjee, S.; Saito,T; Gainaru, C.; Sokolov, A.P. Impact of Hydrogen Bonding on Dynamics of Hydroxyl-Terminated Polydimethylsiloxane. Macromolecules 2016, 49, $3138-3147$. 
(48) Sørensen, T. S.; Compan, V.; Diaz-Calleja, R. Complex permittivity of a film of poly [4(acryloxy)phenyl-(4-chlorophenyl)methanone] containing free ion impurities and the separation of the contributions from interfacial polarization, Maxwell-Wagner-Sillars effects and dielectric relaxations of the polymer chains. J. Chem. Soc.-Faraday Trans. 1996, 92, 1947-1957.

(49) Tadano, K.; Tanaka, Y.; Shimizu, T.; Yano, S. Dielectric Relaxation Studies on Molecular Motion of Poly(fluoroalkyl R-substituted acrylate)s and Compass Motion Model for Internal Motion of the Fluoroalkyl Side Chain. Macromolecules 1999, 32, 1651-1660.

(50) Furukawa, T.; Date, M.; Nakajima, K.; Kosaka, T.; Seo, I. Large dielectric relaxations in an alternate copolymer of vinylidene cyanide and vinyl acetate. Japan. J. Appl. Phys. 1986, 25, 117-1182.

(51) Zou, D.; Iwasaki, S.; Tsutsui, T.; Saito, S.; Kishimoto, M.; Seo, I. Anomaly in dielectric relaxation in alternating copolymers of vinylidene cyanide and fatty acid vinyl ester. Polymer 1990, 31, 1888-1893.

(52) Kahle, S.; Korus, J.; Hempel, E.; Unger, R.; Höring, S.; Schröter, K.; Donth E. GlassTransition Cooperativity Onset in a Series of Random Copolymers Poly(n-butyl methacrylatestat-styrene). Macromolecules 1997, 30, 7214-7223.

(53) Ngai, K. L. Correlation between $\beta$-Relaxation and $\alpha$-Relaxation in the Family of Poly(nbutyl methacrylate-stat-styrene) Random Copolymers. Macromolecules 1999, 32, 7140-7146.

(54) Arous, M.; Kallel, A.; Kaddami, H.; Lahcini, M.; Belfkira, A.; Raihane, M. Copolymerization of a-Cyanovinyl Acetate with Acrylonitrile and Methacrylonitrile: Synthesis, 
Characterization, and Study of their Dielectric Behavior. J. Appl. Polym. Sci. 2009, 114, 10941104.

(55) Hilczer, B.; Malecki, J. Electrets: Studies in Electrical and Electronic Engineering; Elsevier: New York, 1986; Vol. 14, p 19.

(56) Wang, Z.-Y.; Su, K.-H.; Fan, H.-Q.; Wen, Z.-Y. Possible reasons that piezoelectricity has not been found in bulk polymer of polyvinylidene cyanide. Polymer 2008, 49, 2542-2547.

(57) Tasaka, S.; Inagaki, N.; Okutani, T.; Miyata, S. Structure and properties of amorphous piezoelectric vinylidene cyanide copolymers. Polymer 1989, 30, 1639-1642. 


\section{Table of Contents Graphic}

\section{Complex dynamics of a fluorinated vinylidene cyanide copolymer highlighted by dielectric relaxation spectroscopy}

Valter Castelvetro, Simone Capaccioli, Mustapha Raihane,-Atlas Salima

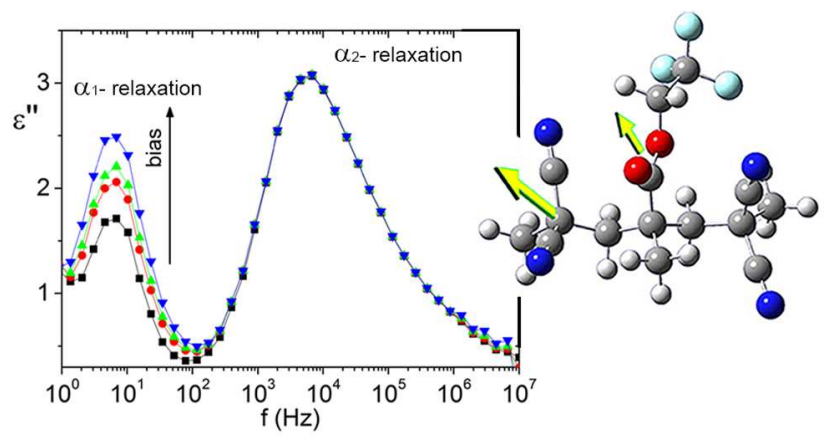


Complex dynamics of a fluorinated vinylidene cyanide copolymer highlighted by dielectric relaxation spectroscopy

Valter Castelvetro, * Simone Capaccioli, Mustapha Raihane, Atlas Salima ToC Graphics $68 \times 35 \mathrm{~mm}(300 \times 300$ DPI $)$ 